\title{
1 Integration of chemosensing and carbon catabolite repression 2 impacts fungal enzyme regulation and plant associations
}

3

4 Wolfgang Hinterdobler ${ }^{1}$, Guofen $\mathrm{Li}^{1}$, David Turrà ${ }^{2, \mathrm{a}}$, Miriam Schalamun ${ }^{1}$, Stefanie Kindel ${ }^{1}$, Ursula Sauer ${ }^{1}$,

5 Sabrina Beier ${ }^{1}$, Aroa Rodriguez Iglesias ${ }^{1}$, Stéphane Compant ${ }^{1}$, Stefania Vitale ${ }^{2}$, Antonio Di Pietro ${ }^{2}$ and

$6 \quad$ Monika Schmoll ${ }^{1 *}$

${ }^{1}$ AIT Austrian Institute of Technology, Center for Health and Bioresources, Tulln, Austria

$9 \quad{ }^{2}$ Departamento de Genetica, Campus de Excelencia Internacional Agroalimentario ceiA3, Universidad de Cordoba, Cordoba, Spain

${ }^{a}$ Current address: Department of Agricultural Sciences, University of Naples Federico II, Portici, Italy.

Running title: Nutrient and plant sensing in T. reesei

Keywords: Trichoderma reesei, chemotropism, plant sensing, sexual development, carbon catabolite

* Corresponding author

Tel: +4366488390594

Email:

21 Wolfgang Hinterdobler

22 Guofen Li

23 David Turra

24 Miriam Schalamun

25 Stefanie Kindel

26 Ursula Sauer

27 Sabrina Beier

28 Aroa Rodriguez-Iglesias

29 Stéphane Compant

30 Stefania Vitale

31 Antonio Di Pietro

32 Monika Schmoll

wolfgang.hinterdobler@ait.ac.at

guofen.li@ait.ac.at

davturra@unina.it

miriam.schalamun@ait.ac.at

stefanie.33@hotmail.com

ursula.sauer@ait.ac.at

sabrina.beier@ait.ac.at

aroa.rguez.iglesias@gmail.com

stephane.compant@ait.ac.at

stefy.vitale@libero.it

ge2dipia@uco.es

monika.schmoll@ait.ac.at 


\section{Abstract}

34

35 Fungal metabolism and enzyme production are regulated by nutrient availability and by interactions with

36 the living environment. We investigated the mechanisms underpinning adaptation of the biotechnological

37 fungus Trichoderma reesei to decaying plant biomass versus living plants. We found that concentration-

38 gated response to glucose, the main molecule sensed from dead plant biomass, is mediated by a conserved

39 signaling pathway downstream of G protein-coupled receptors (GPCRs), while the carbon catabolite

40 repressor CRE1 is critical for glucose concentration gating. The GPCRs CSG1 and CSG2 are further

41 required for root colonization and formation of appressorium like structures on plant surfaces. Acceleration

42 of sexual development in the presence of plant roots and their interactions with fruiting bodies indicates

43 preferential association with plants. Our results reveal a complex sensing network governing resource

44 distribution, enzyme production and fungal development that explains previously observed phenomena in

45 fermentations and opens new perspectives for industrial strain improvement and agriculture. 
48 Sensing of the environment is crucial for all living organisms - be it in their natural habitat or in an industrial

49 setting. Fungi are efficient degraders of plant biomass, and some can also act as root symbionts of living

50 plants by sharing critical nutrients and extending the plant's acquisition network (1-3). The ability to

51 distinguish dead plant material that needs to be enzymatically degraded from a living plant that serves as a

52 mutualistic association partner, is essential to activate the appropriate metabolic and developmental

53 responses during the interaction.

54 Fungi of the genus Trichoderma are ideal models for transfer of natural phenomena to industrial application

55 by studying complex interspecies relationships, as they can act both as saprophytic biomass degraders and

56 plant symbionts $(4,5)$. Trichoderma reesei $(6,7)$ is mostly known as an efficient degrader of cellulosic plant

57 material in nature (8). However, this species was also shown to protect plants against a soil borne oomycete

58 pathogen (9), suggesting that it can undergo beneficial associations with plant roots. Moreover, T. reesei is

59 the only member of the genus in which sexual development has been achieved under laboratory conditions

60 (10), which is significantly regulated by pheromone sensing and external cues (11) and of considerable

61 biotechnological importance for strain improvement $(11,12)$.

62 Fungi can utilize insoluble plant biomass as a source of nutrients by secreting an array of cell wall degrading

63 enzymes (CWDEs) (13). Regulation of CWDE expression in T. reesei has been studied in detail (4, 14).

64 The disaccharide sophorose is thought to act as a natural inducer of cellulolytic enzymes $(15,16)$. However,

65 in the presence of easily metabolized carbon sources such as glucose, CWDE synthesis is inhibited through

66 the carbon catabolite repressor $\operatorname{CRE} 1(17,18)$. Furthermore, regulation of cellulase genes in T. reesei

67 requires conserved components of the heterotrimeric $\mathrm{G}$ protein/cAMP pathway, including the $\mathrm{G}$ protein

68 alpha subunits GNA1 and GNA3, the G protein beta and gamma subunits GNB1 and GNG1, as well as the

69 phosducin like protein PhLP1 (19-23). Recently, two class XIII G protein-coupled receptors (GPCRs),

70 CSG1 and CSG2, were shown to be required for cellulase expression, and CSG1 was also implicated in

71 posttranscriptional regulation of cellulase production (24). 
72 Fungal hyphae have the capacity to chemotropically sense and follow a variety of directional cues, allowing

73 them to successfully locate nutrient sources, mating partners or host organisms (25). Although fungal

74 chemotropism has been known for more than a hundred years (26), the underlying mechanisms and cell

75 signaling pathways remain poorly understood $(25,26)$. Recently, hyphae of the soil-inhabiting plant

76 pathogen Fusarium oxysporum were found to grow towards a variety of carbon and nitrogen sources, as

77 well as towards host plant signals. The chemotropic response to plants is triggered by class III peroxidases

78 released by the roots and requires the fungal peptide pheromone receptor Ste2, as well as a mitogen-activated

79 protein kinase (MAPK) pathway and the NADPH oxidase (NOX) complex (27, 28). While this

80 groundbreaking discovery provided a first glimpse into the complexity of fungal chemosensing, little is

81 known so far on chemotropic signaling in other fungi or on signal integration.

82 Here we report the identification of a chemotropic signaling network in $T$. reesei, which is able to precisely

83 sense glucose concentrations and to accordingly modulate signaling outputs. We further establish that this

84 mechanism allows the fungus to discriminate between dead plant material and living plants and to activate

85 the appropriate metabolic and developmental responses, i.e. enzymatic degradation of biomass for nutrient

86 supply versus root colonization and sexual development.

$88 \quad$ Results

92 Chemotropic growth towards plant exudates has been reported in F. oxysporum (27) and Trichoderma

93 harzianum (29). Here we used a previously described plate chemotropism assay to measure chemotropic

94 sensing in the reference strain QM6a of T. reesei (Figure 1a). Because QM6a does not readily germinate in

95 water agar, $0.0025 \% \mathrm{w} / \mathrm{v}$ peptone was added to the agar. This concentration was optimized to ensure

96 coordinated conidial germination above $60 \%$ without interfering with chemotropism. As previously

97 reported for F. oxysporum (27), T. reesei exhibited a significant chemotropic response to $1 \%$ (w/v) glucose 
and $5 \%(\mathrm{w} / \mathrm{v})$ sodium glutamate (Figure $1 \mathrm{~b})$. These results confirm that the optimized assay conditions are appropriate for measuring the chemotropic response of $T$. reesei to different carbon and nitrogen sources.

CWDE expression in T. reesei is tightly regulated to trigger production of the appropriate enzyme cocktail

for degradation of the different cell wall materials (15). We therefore asked whether this fungus can chemotropically sense building blocks of the plant cell wall. None of the high molecular weight compounds cellulose, xylan and pectin elicited a significant chemotropic response, most likely due to the insoluble nature of these polymers (Figure 1c). In line with this idea, cellulose and pectin did induce a significant chemotropic response after autoclaving (Figure S1a), and this response was abolished when the soluble compounds released by autoclaving were washed away prior to the chemotropism assay (see below). Hyphal chemotropism of $T$. reesei is thus elicited by soluble degradation products of cellulose and pectin, as previously reported in F. oxysporum (27).

We next measured the chemotropic response of QM6a to different glucose concentrations ranging from limiting levels $(0.0625 \% \mathrm{w} / \mathrm{v})$ to very high concentrations (5\%) generating considerable osmotic pressure.

We conclude that glucose chemosensing in $T$. reesei is subject to gating, allowing for an optimum 119 chemotropic response in a limited range (gate) of concentrations.

120 We next tested additional low molecular weight carbon sources released upon plant cell wall degradation or 121 involved in enzyme regulation, including glycerol, cellobiose or lactose, the transglycosylation product 122 sophorose, as well as to D-xylose, D-galactose or L-arabinose, all of which act as inducers or repressors of 123 CWDEs $(16,32)$. However, none of these compounds elicited a significant chemotropic response when 
124 applied at $55 \mathrm{mM}$, which provided the optimum response for glucose (Figure 1c). Based on these results,

125 we conclude that glucose is the main soluble compound released from degradable plant material eliciting

126 chemotropic hyphal growth in T. reesei. Nevertheless, these other plant cell wall derived compounds may

127 still be sensed by the fungus and cause physiological responses other than chemotropism.

Glucose chemosensing requires the GPCRs CSG1/CSG2 and functions via the heterotrimeric G proteincAMP-PKA pathway

We next asked how chemotropic sensing of glucose is mediated in T. reesei. Previous studies suggested that the two class XIII GPCRs CSG1 and CSG2, which are involved in regulation of cellulase gene expression, could act as cellulose or glucose-sensors (24). However, the precise function of these GPCRs in glucose signaling has not been addressed. Here we found that the chemotropic response to all glucose concentrations tested was largely abolished in the $\Delta c \operatorname{cs} 1$ and $\Delta c s g 2$ single and double mutants (Figure 1e, f). By contrast, mutants in two other GPCR genes, $\Delta 4508$ and $\Delta 80125$, were unaffected in their response, thus confirming the specificity of CSG1 and CSG2 in glucose chemosensing (Figure S1d) and that the $h p h$ selection marker does not interfere with chemotropic responses. Similar to the wild type strain, the $\Delta \operatorname{csg} 1$ and $\Delta \operatorname{csg} 2$ mutants failed to show a significant response to the other low molecular weight carbon sources tested (Figure S2). has differential impacts on cellulase regulation (24). were previously shown to function in cellulase regulation (Figure 2a). Here we found that the chemotropic response to $1 \%$ glucose was abolished in $T$. reesei deletion mutants lacking either the G alpha subunits GNA1, GNA2, or GNA3, the G beta subunit GNB1, the G gamma subunit GNG1, the phosducin PhLP1, 148 the catalytic subunit of protein kinase A, PKAc1, or the adenylate cyclase ACY1 (Figure 2 a, b). 149 Interestingly, constitutive activation of GNA1 and GNA3 $(19,20)$, but not of GNA2, also abolished the 
Although $T$. reesei is mostly known as an efficient degrader of dead plant material (8), this fungus has been suggested to undergo beneficial associations with plant roots (9). We therefore asked whether T. reesei is able to chemotropically sense the presence of the living plant. In the plant pathogen $F$. oxysporum, chemotropic growth towards roots is triggered by secreted class III plant peroxidases and requires the GPCR Ste2, the cognate receptors of peptide sex pheromone alpha $(27,30)$. Here we found that similar to $F$. oxysporum, T. reesei exhibited a significant chemotropic response to root exudates from soybean or tomato plants (Figure 2d), as well as a concentration dependent response to commercial horse radish peroxidase (HRP) with a peak at $8 \mu \mathrm{M}$ (Figure 2e). Both responses required the GPCR orthologues of Ste2 and Ste3, which are named HPR1 and HPR2 in T. reesei (Figure 2f). Importantly, HPR1 and HPR2 were also required reinforcing the functional link between sexual development and plant recognition in fungi. heterotrimeric $\mathrm{G}$ protein-cAMP-PKA pathway are also required for chemosensing of living plant roots. In contrast to the wild type strain, the $\Delta \operatorname{csg} 1$ and $\Delta c s g 2$ single and double mutants lacked a chemotropic response to root exudates (Figure 2g). Likewise, mutants in GNA1, GNA2, GNA3, PKAc1 or ACY1 were

173 impaired in directed growth towards the chemoattractants released from plant roots (Figure 2h). Taken 174 together, these results indicate that chemosensing of plant signals by T. reesei depends on the sex pheromone 175 receptors as well as on the GPCRs CSG1 and CSG2, which act upstream of the G protein/cAMP pathway. 
178 Due to the importance of glucose as a signaling molecule released from plant biomass, we next investigated

179 the role of carbon catabolite repression (CCR), a conserved regulatory mechanism that prevents unnecessary

180 production of fungal CWDEs in the presence of readily utilizable carbon sources such as glucose (18).

181 Similar to other fungi, CCR in T. reesei is mediated by the conserved carbon catabolite repressor CRE1

182 (17). Although the chemotropic response to glucose was still functional in a $\Delta$ crel mutant, the optimum

183 response concentration was dramatically shifted to $5 \%$, as compared to $1 \%$ in the wild type strain (Figure

184 2i). At a higher concentration of $7.5 \%$, a decrease in the response was observed, suggesting that

185 concentration dependence is still functional in the $\Delta c r e 1$ mutant. Most importantly, the $\Delta c r e 1$ mutant was

186 also impaired in the response to plant root exudates (Figure 2g). Thus, CRE1-mediated carbon catabolite

187 repression regulates the sensitivity of chemoperception and impacts gating of the chemotropic response both

188 to glucose and to plant root exudates.

Chemosensing via CSG1/CSG2 impacts hyphal morphology on cellulosic substrate as well as root colonization

In nature, nutrient sensing is crucial to induce adaptive responses to the substrate. For example, some whether chemosensing through CSG1 and CSG2 impacts morphogenetic development of $T$. reesei in response to dead and living plant substrates. Scanning electron microscopy revealed the presence of characteristic appressorium-like attachment structures at the tips of the wild type hyphae growing on surface-sterilized maple leaves (Figure 3a). Similar structures were also observed during growth on cellophane membranes (Figure S4). However, no such structures were detected in the mutants lacking either 
We next tested the ability of the different strains to colonize living soybean roots. While the wild type strain roots.

Because nutrient availability is crucial for efficient sexual development (35), we wondered whether glucose sensing by CSG1/CSG2 impacts sexual development and/or chemical communication by secretion of secondary metabolites(36) prior to mating. Here we found that the mating behavior of the mutants lacking

CSG1 and/or CSG2 was not significantly changed compared to wild type and that both showed altered pigments) (Figure S5a-h).

Our finding that chemotropic growth of T. reesei towards roots requires the Ste2 and Ste3 orthologs HPR1 and HPR2 confirms earlier results showing that chemotropic sensing of the host plant functions via a sex pheromone receptor $(27,30)$. We therefore asked whether plant signals impact sexual development of $T$. reesei. To this aim, mating experiments were performed in the absence or presence of soybean seedlings, using two different combinations of opposing mating types: strains CBS999.97 MAT1-1 x CBS999.97

223 MAT1-2 and FF1 x FF2, two female fertile derivatives of QM6a. In both mating assays, fruiting body

224 formation was accelerated by one day in the presence of soybean seedlings (day 6 vs. day 7 in control 225 conditions) (Figure S5g and Figure S6, 7). This effect was only observed in the physical presence of the 226 plant, but not when plant root exudates were added to the medium. Moreover, accelerated fruiting body 227 formation was not mimicked by the presence of a wooden toothpick or a glass rod, suggesting that it is not 

seedlings (Figure S5g). By contrast, as reported previously, fruiting body formation in a $\Delta h p r 1 \Delta h p r 2$ double receptor mutant was impaired and ascospore formation was abolished in the single receptor mutants of their cognate mating type (37). These defects were not rescued by the presence of the plant (Figure S7, red background). We conclude that interaction with the plant stimulates fruiting body formation in $T$. reesei but cannot substitute for the requirement of the pheromone-receptor interaction.

To further explore the effect of the living plant on fungal mating, we conducted microscopic analysis of attached to the root surface and contained morphologically normal perithecia (Figure $4 b-n$ ). We further observed a thickening of the root around the contact zone with the fruiting body and could clearly discern an interaction zone, where hyphae extended towards the root without extensively penetrating it (Figure 4e,

249 (Figure 4 d,e). Viability staining with methylene blue highlighted a region of the fruiting body mycelium 250 surrounding the root canal, indicative of a response of the fungal mycelium to the plant (Figure 4e (4)). A 251 striking response observed was the de novo formation of fruiting body tissue in the presence of the root 252 (Figure 4b, g-i). Roots enclosed inside such a structure displayed signs of degradation, possibly as a result 253 of plant or fungal defense reactions (Figure 4l-n ). We next asked whether the absence of the fungal 
254 pheromone receptor would result in increased damage or growth reduction of the plant roots, but found no 255 evidence for this (supplementary note 3, Figure S10). We conclude that fruiting bodies of T. reesei respond morphologically to presence of plant roots, which may act both as a carbon source as well as a stimulus for

257 sexual development (see supplementary note 4).

\section{Discussion}

Every single spot in nature is teeming with life, with diverse organisms competing or living in synergy. The natural habitat of Trichoderma are forests, where decaying plant material coexists with living plants (1). In this complex environment, the fungus must adapt its lifestyle to different necessities. Here we provide evidence showing that $T$. reesei integrates chemosensing of glucose monomers released from cellulosic plant biomass with signals from living plants, via the GPCRs CSG1/CSG2 and the conserved heterotrimeric G protein-cAMP-PKA signaling cascade, to efficiently adjust its responses to the surrounding nutritional and "social" landscape. To achieve this adaptation, our data indicate that T. reesei uses feedback cycles of CDWE release, sensing of degradation products such as glucose or sophorose, and subsequent adjustment of CDWE regulation (Figure 5). While the regulatory mechanisms controlling expression of CWDEs, sexual development and plant interactions in Trichoderma spp. have been studied in detail $(4,5,11,14)$, the biological relevance and the interconnections between the different pathways have remained elusive.

272 Previous work indicated that CSG1 is crucial for posttranscriptional regulation of cellulase gene expression

273 (24), but not for induction of transcription. We show that CSG1 senses a defined concentration of glucose,

274 and that a specific signal level transmitted through the G-protein cascade is required for the chemotropic

275 response. Consequently, sensing of this specific glucose concentration must be the signal required for 276 initiation of enzyme production after transcriptional induction by sophorose and hence represents a second 277 checkpoint. This explains why constitutive activation of $\mathrm{G}$ alpha subunits $(19,20)$, which, as shown here, 278 are crucial for glucose signal transmission, does not result in inducer-independent cellulase production: 279 without transcriptional induction (first checkpoint), glucose levels sensed by this pathway are not relevant. 
280 Thus we propose that, in agreement with previous work (24), chemosensing of glucose concentration 281 represents a crucial checkpoint for efficient utilization of cellulosic substrates and establishment of a 282 physical interaction with plant roots.

283 We further show that the carbon catabolite repressor CRE1 is important for concentration dependent glucose 284 sensing. CRE1 transcript levels (38) and nuclear localization (39) were previously shown to be regulated by 285 glucose concentrations. Our results are in line with previous findings showing that deletion of crel alleviates 286 the requirement for nutrient limitation to produce high enzyme levels in fermentors (40-42). Reminiscent of 287 the "gating" found here for the chemotropic response to glucose, cAMP was shown to stimulate cellulase 288 gene expression in $T$. reesei only at certain concentrations (23). Moreover, in yeasts glucose sensing and 289 signaling was previously linked to the cAMP-PKA pathway $(43,44)$. Here we provide further support for 290 the crucial role of the adenylate cyclase ACY1 and PKAc1, together with CRE1, in concentration dependent 291 glucose signaling, which is likely to be crucial for improvements towards reliable upscaling of 292 fermentations.

293 Degradation of dead plant material by fungal CWDEs yields increasing amounts of glucose, which are in 294 balance with the level of CWDE gene expression. Our results suggest the presence of a negative feedback 295 mechanism which is controlled via the fine-tuned sensing of glucose levels, in parallel to the detection of 296 the inducer. This finding is biologically highly relevant, because living plant roots are known to secrete 297 sugars (45) and exuded glucose could thus act as an inducing signal for root colonization by fungi $(46,47)$ 298 in which transmission of nutrients to a fungal interaction partner is tightly regulated and requires contact $299(48,49)$. Such a chemotropic signaling circuit should act separately from that controlling CWDE production 300 to allow differentiation between dead litter and living plants acting as a "live nutrient source". We propose 301 that $T$. reesei distinguishes between live and dead plant material by sensing the balance of secreted CDWEs 302 and the amount of glucose released by these enzymes. Combined with our findings that root signals are 303 sensed via two different pathways - the pheromone receptors and the glucose-sending GPCRs CSG1/2, and 304 that plant roots stimulate sexual development and interaction with fungal fruiting bodies, we propose that 305 T. reesei applies a bipartite checkpoint mechanism involving glucose sensing via the heterotrimeric $\mathrm{G}$ 
protein pathway and the peroxidase signals detected by sex pheromone receptors. This mechanism is likely

to enable $T$. reesei to optimize resource efficiency by tight regulation of CWDE biosynthesis versus plant by crossing and exploration of fungal symbiosis or pathogenesis of plants.

\section{Methods}

Strains and cultivation conditions

For analysis of mating responses, recombinant strains in the fully fertile background of CBS999.97 in both mating types were used $(10,37)$. Assessment of chemotropic responses to nutrients were performed with wild type strains and mutants in the QM6a (6), QM9414 (50), TU-6 (51) or CBS999.97 strain backgrounds, depending on the mutants used, in order to be able to evaluate the results against the scientific background on regulation of plant cell wall degradation. Strains FF1 or FF2 (fully fertile derivative strains of QM6a; mating type MAT1-1 (FF1) or MAT1-2 (FF2)) were used for preparation of double mutants of selected genes (52). Strains $\Delta 4508$ and $\Delta 80125$ were constructed as described previously (53) and deletions were used in this study is shown in Table S1.

For all chemotropic assays, strains were grown on malt extract agar $(3 \% \mathrm{w} / \mathrm{v})$ at $28{ }^{\circ} \mathrm{C}$ until sporulation $(4$ days) prior to harvesting of conidia. For sexual development, compatible strains were cultivated on malt extract agar $(2 \% \mathrm{w} / \mathrm{v})$ at room temperature in daylight (light-dark cycles) until fruiting body formation and subsequent ascospore discharge (55). For analysis of the relevance of soybean seedlings for sexual 
were subsequently incubated on malt extract agar plates. Germinating soybeans were placed onto mating cultures prior to inoculation of fungal mating partners. All chemicals used were supplied by Roth (Karlsruhe,

334 Germany) unless stated otherwise.

338 The mutants $\Delta c s g 1$ and $\Delta c s g 2$ in the QM6a background (24) were crossed with strain FF1 and ascospores 339 were harvested. Single spores were isolated and tested for the presence of the mutations by PCR. Mating 340 types were analyzed as described previously (10). The resulting progeny for both mutants in female fertile background were crossed to obtain $\Delta \operatorname{csg} 1 \Delta \operatorname{csg} 2$ double mutants.

\section{Preparation of chemotropic agents and plant root exudates}

345 For carbon sources, compounds were dissolved in purified water to the respective concentration and filter

346 sterilized. Insoluble carbon sources were autoclaved and subsequently washed to remove degradation 347 products released by autoclaving.

348 For preparation of plant root exudates, soybeans were surface sterilized (as described above) and planted in 349 sterilized perlite (premium perlite 2-6, Gramoflor GmbH, Germany). Plants were grown until the second 350 leaf stage and recovered from the perlite. After gentle washing with water, plant roots were carefully 351 submerged in sterile water and kept for 2 days at room temperature. Root exudates were filter sterilized and 352 stored at $-80{ }^{\circ} \mathrm{C}$.

356 Chemotropism plate assays were done essentially as described (27). Briefly, fresh conidia harvested from 357 4-day-old plates were resuspended in $1 \mathrm{~mL}$ spore solution $(0.8 \%(\mathrm{w} / \mathrm{v}) \mathrm{NaCl}$ and $0.05 \%(\mathrm{w} / \mathrm{v})$ Tween 80$)$. 
358 The suspension was filtered through glass wool, centrifuged at $8000 \mathrm{rpm}$ for 2 minutes, the supernatant was

359 discarded, and the pellet was resuspended in $1 \mathrm{~mL}$ of sterile purified water. The suspension was applied at 360 a concentration of $10^{8}$ conidia per $\mathrm{mL}$ to assay plates containing water agar $(0.5 \%$ w/v; Roth, Karlsruhe, 361 Germany No 5210.2). Different concentrations of peptone (from casein; Merck, Darmstadt, Germany; No 362 1.11931) were added to facilitate germination. The optimal concentration was determined to be $0.0025 \%$ 363 (w/v) peptone. For the uridine auxotropic strain TU-6, $10 \mu \mathrm{M}$ uridine was added to the medium, when used 364 as wild-type control. After application of the chemoattractant and the control solution, plates were incubated 365 at $28^{\circ} \mathrm{C}$ in darkness. After 13 hours of incubation, orientation of germ tubes was determined microscopically 366 (VisiScope TL524P microscope; 200x magnification) and chemotropic indices were calculated as described 367 earlier (27).

\section{Statistics}

Statistic evaluation of results was performed using Student's T-Test (two-sided) in R Studio (compare

372 means, ggpubr v0.3.0). Background in variations of hyphal orientation of wild type strains in the absence 373 of a chemoattractant was used as control for statistical evaluation (10 biological replicates).

377 Patterns of secreted metabolites were analyzed by excising agar slices close to the contact zone from plates 378 with fungi grown under the conditions described. Three biological replicates of three plates pooled each 379 were used for analysis as described previously by HPTLC (high performance thin layer chromatography) 380 (56). Briefly, agar slices were extracted with chloroform and acetone, evaporated and resuspended in 381 chloroform. The samples were spotted on TLC plates (HPTLC silica gel 60 F254s) using the CAMAG 382 Automatic TLC sampler 4 (CAMAG, Muttenz, Switzerland). After separation, plates were analyzed at 383 different wave lengths using the CAMAG visualizer (CAMAG). 
Soybean seeds were surface sterilized and rinsed three times with PBS (phosphate buffered saline, pH7.2).

Seeds were then put onto PDA plates containing T. reesei strains. Thereafter, the seeds were replaced into sterile magenta boxes containing twice autoclaved soil (1:1:1 perlite, sand, potting soil) and sterilized tap water $(25 \mathrm{~mL})$. After 10 days of incubation in the greenhouse, plants were harvested, immersed in PBS (15 $\mathrm{mL}$ ) containing $50 \mu \mathrm{g} / \mathrm{mL}$ of wheat germ agglutinin (WGA)-AlexaFluor488 conjugate (Life Technologies, USA). Plant colonization was analyzed after 2 hours of incubation with (WGA)-Alexa Fluor488 at $37{ }^{\circ} \mathrm{C}$ and again rinsing three times with PBS.

Microscopy was performed using a confocal microscope (Olympus Fluoview FV1000 with multi-line laser

FV5-LAMAR-2 and HeNe(G)laser FV10-LAHEG230-2, Japan) with objectives of 10x, 20x and 40x magnification. X, Y, Z pictures and scans were taken at 405, 488 and $549 \mathrm{~nm}$ with same settings and normal light. The software Imaris (Bitplane, Zürich, Switzerland) was used for visualization. ImageJ software $(1.47 \mathrm{v})$ was applied to merge pictures from different channels. 


\section{Author contributions}

WH contributed to chemotropic analysis, performed microscopy and secondary metabolite analysis, participated in designing figures, performed statistical analysis of data and contributed to drafting and editing the manuscript. GL contributed to chemotropic analysis, growth assays and performed confocal microscopy under the supervision of SC. SC supervised work and designed pictures from confocal microscopy. SK and US performed electron microscopy. MiS and SB contributed to chemotropic analysis and SB further supported plant assays. ARI contributed construction of the strain with constitutive activation of GNA2. SV provided preliminary data and contributed to conception of the study. DT and ADP supervised part of the work of GL, participated in conception of the study, interpretation and editing of the final manuscript. MS conceived the study, supervised students, contributed to analysis of results, interpreted data, designed figures and wrote the final version of the manuscript.

\section{Acknowledgements}

We want to thank Viktoria Fabsits for technical assistance and Stefan Böhmdorfer (University of Natural 


\section{Competing interests}

The authors declare no competing interests.

\section{REFERENCES}

1. Harman GE, Howell CR, Viterbo A, Chet I, \& Lorito M (2004) Trichoderma species-opportunistic, avirulent plant symbionts. Nat Rev Microbiol 2(1):43-56.

2. Mäkelä MR, Donofrio N, \& de Vries RP (2014) Plant biomass degradation by fungi. Fungal Genet Biol 72:2-9.

3. Rasmann S, Bennett A, Biere A, Karley A, \& Guerrieri E (2017) Root symbionts: Powerful drivers of plant above- and belowground indirect defenses. Insect Sci 24(6):947-960.

4. Bischof RH, Ramoni J, \& Seiboth B (2016) Cellulases and beyond: the first 70 years of the enzyme producer Trichoderma reesei. Microb Cell Fact 15(1):106.

5. Guzman-Guzman P, Porras-Troncoso MD, Olmedo-Monfil V, \& Herrera-Estrella A (2019) Trichoderma species: Versatile plant symbionts. Phytopathology 109(1):6-16.

6. Martinez D, et al. (2008) Genome sequencing and analysis of the biomass-degrading fungus Trichoderma reesei (syn. Hypocrea jecorina). Nat Biotechnol 26(5):553-560.

7. Schmoll M, et al. (2016) The genomes of three uneven siblings: footprints of the lifestyles of three Trichoderma species. Microbiol Mol Biol Rev 80(1):205-327.

8. Schmoll M, Seiboth B, Druzhinina I, \& Kubicek CP (2014) Genomics analysis of biocontrol species and industrial enzyme producers from the genus Trichoderma. The Mycota XIII, eds Esser K \& Nowrousian M (Springer, Berlin), pp 233-266.

9. Seidl V, et al. (2006) Antagonism of Pythium blight of zucchini by Hypocrea jecorina does not require cellulase gene expression but is improved by carbon catabolite derepression. FEMS Microbiol Lett 257(1):145-151.

10. Seidl V, Seibel C, Kubicek CP, \& Schmoll M (2009) Sexual development in the industrial workhorse Trichoderma reesei. Proc Natl Acad Sci U S A 106(33):13909-13914.

11. Hinterdobler W, Beier S, S. K, \& Schmoll M (2020) Sexual development, its determinants and regulation in Trichoderma reesei. Recent Developments in Trichoderma Research, New and future developments in microbial biotechnology and bioengineering, eds Zeilinger S, Druzhinina I, Singh HB, \& Gupta VK (Elsevier, Amsterdam, The Netherlands), pp 185-206.

12. Hinterdobler W, et al. (2021) Trichoderma reesei isolated from Austrian soil with high potential for biotechnological application. Front Microbiol 12:552301.

13. Glass NL, Schmoll M, Cate JH, \& Coradetti S (2013) Plant cell wall deconstruction by ascomycete fungi. Annu Rev Microbiol 67:477-498.

14. Schmoll M (2018) Regulation of plant cell wall degradation by light in Trichoderma. Fungal Biol Biotechnol 5:10.

15. Foreman PK, et al. (2003) Transcriptional regulation of biomass-degrading enzymes in the filamentous fungus Trichoderma reesei. J Biol Chem 278(34):31988-31997.

16. Bazafkan H, Tisch D, \& Schmoll M (2014) Regulation of glycoside hydrolase expression in Trichoderma. Biotechnology and Biology of Trichoderma, eds Gupta VK, Schmoll M, HerreraEstrella A, Upadhyay RS, Druzhinina I, \& Tuohy MG (Elsevier, Oxford, UK), pp 291-307.

17. Strauss J, et al. (1995) Cre1, the carbon catabolite repressor protein from Trichoderma reesei. FEBS Lett 376(1-2):103-107.

18. Adnan M, et al. (2017) Carbon catabolite repression in filamentous fungi. Int J Mol Sci 19(1).

19. Schmoll M, Schuster A, do Nascimento Silva R, \& Kubicek CP (2009) The G-alpha protein GNA3 of Hypocrea jecorina (anamorph Trichoderma reesei) regulates cellulase gene expression in the presence of light. Eukaryot Cell 8(3):410 - 420. 
20. Seibel C, et al. (2009) Light-dependent roles of the G-protein subunit GNA1 of Hypocrea jecorina (anamorph Trichoderma reesei). BMC Biol 7(1):58.

21. Tisch D, Kubicek CP, \& Schmoll M (2011) The phosducin-like protein PhLP1 impacts regulation of glycoside hydrolases and light response in Trichoderma reesei. BMC Genomics 12:613.

22. Schuster A, Tisch D, Seidl-Seiboth V, Kubicek CP, \& Schmoll M (2012) Roles of protein kinase A and adenylate cyclase in light-modulated cellulase regulation in Trichoderma reesei. Appl Environ Microbiol 78(7):2168-2178.

23. Sestak S \& Farkas V (1993) Metabolic regulation of endoglucanase synthesis in Trichoderma reesei: participation of cyclic AMP and glucose-6-phosphate. Can J Microbiol 39(3):342-347.

24. Stappler E, Dattenböck C, Tisch D, \& Schmoll M (2017) Analysis of light- and carbon-specific transcriptomes implicates a class of G-protein-coupled receptors in cellulose sensing. mSphere 2(3):e00089-00017.

25. Brand A \& Gow NA (2009) Mechanisms of hypha orientation of fungi. Curr Opin Microbiol 12(4):350-357.

26. Turra D, Nordzieke D, Vitale S, El Ghalid M, \& Di Pietro A (2016) Hyphal chemotropism in fungal pathogenicity. Semin Cell Dev Biol 57:69-75.

27. Turra D, El Ghalid M, Rossi F, \& Di Pietro A (2015) Fungal pathogen uses sex pheromone receptor for chemotropic sensing of host plant signals. Nature 527(7579):521-524.

28. Nordzieke DE, Fernandes TR, El Ghalid M, Turra D, \& Di Pietro A (2019) NADPH oxidase regulates chemotropic growth of the fungal pathogen Fusarium oxysporum towards the host plant. New Phytol 224(4):1600-1612.

29. Lombardi N, et al. (2018) Root exudates of stressed plants stimulate and attract Trichoderma soil fungi. Mol Plant Microbe Interact.

30. Vitale S, Di Pietro A, \& Turra D (2019) Autocrine pheromone signalling regulates community behaviour in the fungal pathogen Fusarium oxysporum. Nat Microbiol 4(9):1443-1449.

31. Vitale S, et al. (2017) Structure-activity relationship of alpha mating pheromone from the fungal pathogen Fusarium oxysporum. J Biol Chem 292(9):3591-3602.

32. Margolles-Clark E, Ilmén M, \& Penttilä M (1997) Expression patterns of ten hemicellulase genes of the filamentous fungus Trichoderma reesei on various carbon sources. J Biotechnol 57:167179.

33. Cortes C, et al. (1998) The expression of genes involved in parasitism by Trichoderma harzianum is triggered by a diffusible factor. Mol Gen Genet 260(2-3):218-225.

34. Elad Y, Chet I, Boyle P, \& Henis Y (1983) Parasitism of Trichoderma spp. on Rhizoctonia solani and Sclerotium rolfsii - Scanning Electron Microscopy and Fluorescence Microscopy. Phytopathology 73(1):85-88.

35. Debuchy R, Berteaux-Lecellier V, \& Silar P (2010) Mating systems and sexual morphogenesis in ascomycetes. Cellular and Molecular Biology of Filamentous Fungi, eds Borkovich KA \& Ebbole DJ (ASM Press, Washington, DC), pp 501 - 535.

36. Bazafkan H, et al. (2015) Mating type dependent partner sensing as mediated by VEL1 in Trichoderma reesei. Mol Microbiol 96(6):1103-1118.

37. Seibel C, Tisch D, Kubicek CP, \& Schmoll M (2012) The role of pheromone receptors for communication and mating in Hypocrea jecorina (Trichoderma reesei). Fungal Genet Biol 49(10):814-824.

38. Jekosch K \& Kück U (2000) Glucose dependent transcriptional expression of the crel gene in Acremonium chrysogenum strains showing different levels of cephalosporin $\mathrm{C}$ production. Curr Genet 37(6):388-395.

39. Lichius A, Seidl-Seiboth V, Seiboth B, \& Kubicek CP (2014) Nucleo-cytoplasmic shuttling dynamics of the transcriptional regulators XYR1 and CRE1 under conditions of cellulase and xylanase gene expression in Trichoderma reesei. Mol Microbiol.

40. Nakari-Setälä T, et al. (2009) Genetic modification of carbon catabolite repression in Trichoderma reesei for improved protein production. Appl Environ Microbiol 75(14):4853-4860. 
41. Arvas M, et al. (2011) Correlation of gene expression and protein production rate - a system wide study. BMC Genomics 12:616.

42. Rassinger A, Gacek-Matthews A, Strauss J, Mach RL, \& Mach-Aigner AR (2018) Truncation of the transcriptional repressor protein Cre1 in Trichoderma reesei Rut-C30 turns it into an activator. Fungal Biol Biotechnol 5:15.

43. Conrad M, et al. (2014) Nutrient sensing and signaling in the yeast Saccharomyces cerevisiae. FEMS Microbiol Rev 38(2):254-299.

44. Van Ende M, Wijnants S, \& Van Dijck P (2019) Sugar sensing and signaling in Candida albicans and Candida glabrata. Front Microbiol 10:99.

45. Timotiwu PB \& Sakurai N (2002) Identification of mono-, oligo-, and polysaccharides secreted from soybean roots. J Plant Res 115(1118):77-85.

46. Hermosa R, Viterbo A, Chet I, \& Monte E (2012) Plant-beneficial effects of Trichoderma and of its genes. Microbiology-Sgm 158:17-25.

47. Vargas WA, Mandawe JC, \& Kenerley CM (2009) Plant-derived sucrose is a key element in the symbiotic association between Trichoderma virens and maize plants. Plant Physiol 151(2):792808.

48. Bever JD, Richardson SC, Lawrence BM, Holmes J, \& Watson M (2009) Preferential allocation to beneficial symbiont with spatial structure maintains mycorrhizal mutualism. Ecol Lett 12(1):1321.

49. Werner GD \& Kiers ET (2015) Partner selection in the mycorrhizal mutualism. New Phytol 205(4):1437-1442.

50. Vitikainen M, et al. (2010) Array comparative genomic hybridization analysis of Trichoderma reesei strains with enhanced cellulase production properties. BMC Genomics 11:441.

51. Gruber F, Visser J, Kubicek CP, \& de Graaff LH (1990) The development of a heterologous transformation system for the cellulolytic fungus Trichoderma reesei based on a pyrG-negative mutant strain. Curr Genet 18(1):71-76.

52. Tisch D, et al. (2017) Omics Analyses of Trichoderma reesei CBS999.97 and QM6a indicate the relevance of female fertility to carbohydrate-active enzyme and transporter levels. Appl Environ Microbiol 83(22).

53. Schuster A, et al. (2012) A versatile toolkit for high throughput functional genomics with Trichoderma reesei. Biotechnol Biofuels 5(1):1.

54. Tronsmo A (1991) Biological and integrated controls of Botrytis cinerea on apple by Trichoderma harzianum. Biological Control 1(1):59-62.

55. Schmoll M (2013) Sexual development in Trichoderma - scrutinizing the aspired phenomenon. Trichoderma: Biology and Applications, eds Mukherjee PK, Horwitz BA, Singh US, Mukherjee M, \& Schmoll M (CAB International, UK), pp 67-86.

56. Hinterdobler W, et al. (2019) The role of PKAc1 in gene regulation and trichodimerol production in Trichoderma reesei. Fungal Biol Biotechnol 6:12. 
a
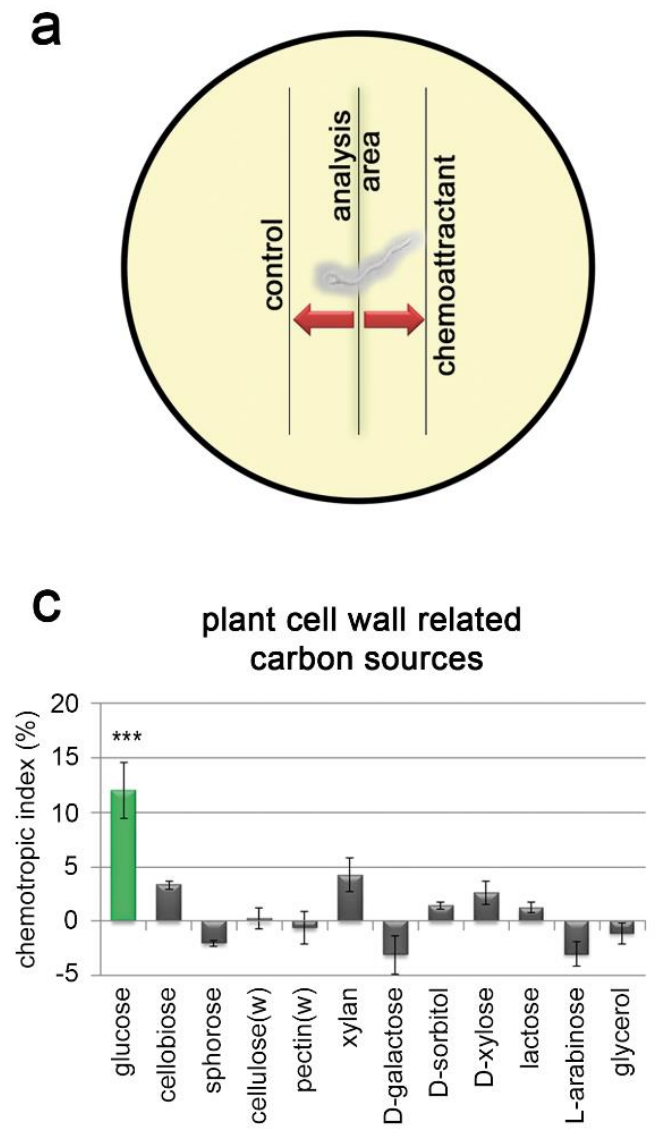

e

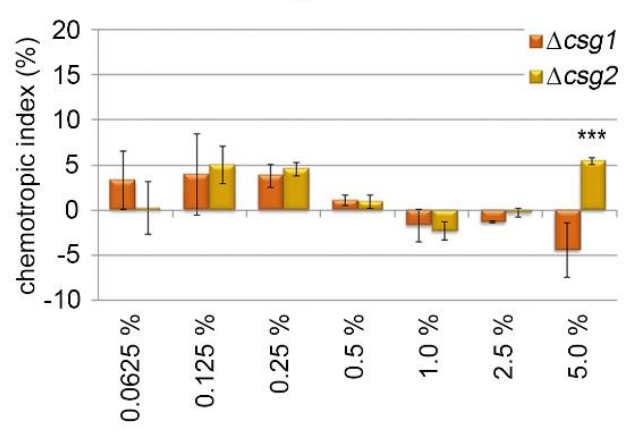

b
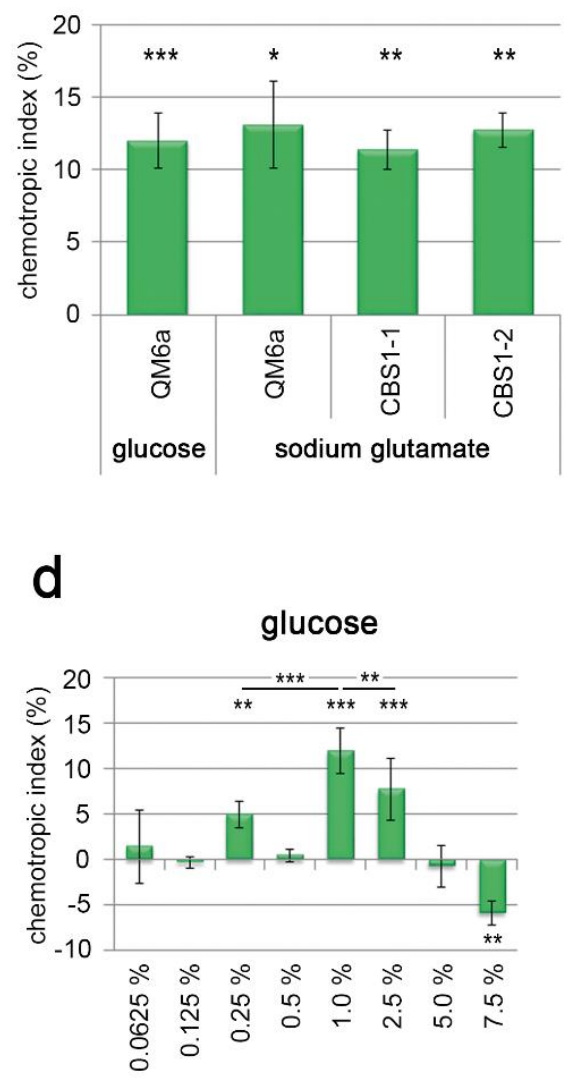

f

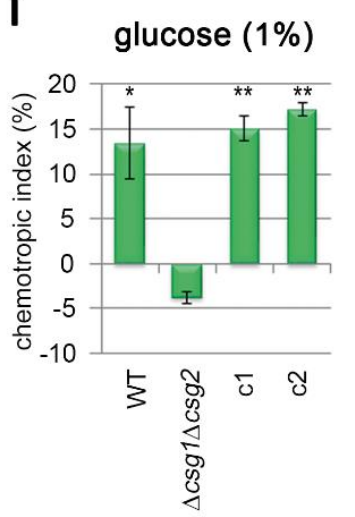

Figure 1. T. reesei responds chemotropically to nutrients. (a) Schematic representation of the experimental setup for analysis of chemotropic responses. (b) Chemotropic response of different $T$. reesei wild type strains to $1 \%$ glucose or $5 \%$ sodium glutamate. (c) Chemotropic response of wild type strain QM6a to different carbon sources related to degradation of plant biomass. Soluble carbon sources were used at $55 \mathrm{mM}$ corresponding to $1 \%$ glucose, cellulose and pectin were used at $1 \%$ and washed ("w") after autoclaving prior to application. (d, e) Chemotropic response of QM6a (d) or the G protein-coupled receptor mutants $\Delta \operatorname{csg} 1$ and $\Delta \operatorname{csg} 2$ (e) to different glucose concentrations. (f) Chemotropic response of a $\Delta \operatorname{csg} 1 \Delta \operatorname{csg} 2$ double mutant and two progeny from the crossings of QM6a $\Delta \operatorname{csg} 1$ and QM6a $\Delta \operatorname{csg} 2$ with FF1, in which these deletions are restored ( $\mathrm{c} 1$ and $\mathrm{c} 2$ ). Error bars show standard deviations from at least two biological replicates. Asterisks mark statistical significance of chemotropism in comparison to the control (absence of a chemoattractant). Statistical significance between tested strains or concentrations is indicated by asterisks over black bars. $*$ p-value $<0.1, * *$ p-value $<0.05$ and $* * *$ p-value $<0.01$. 
a
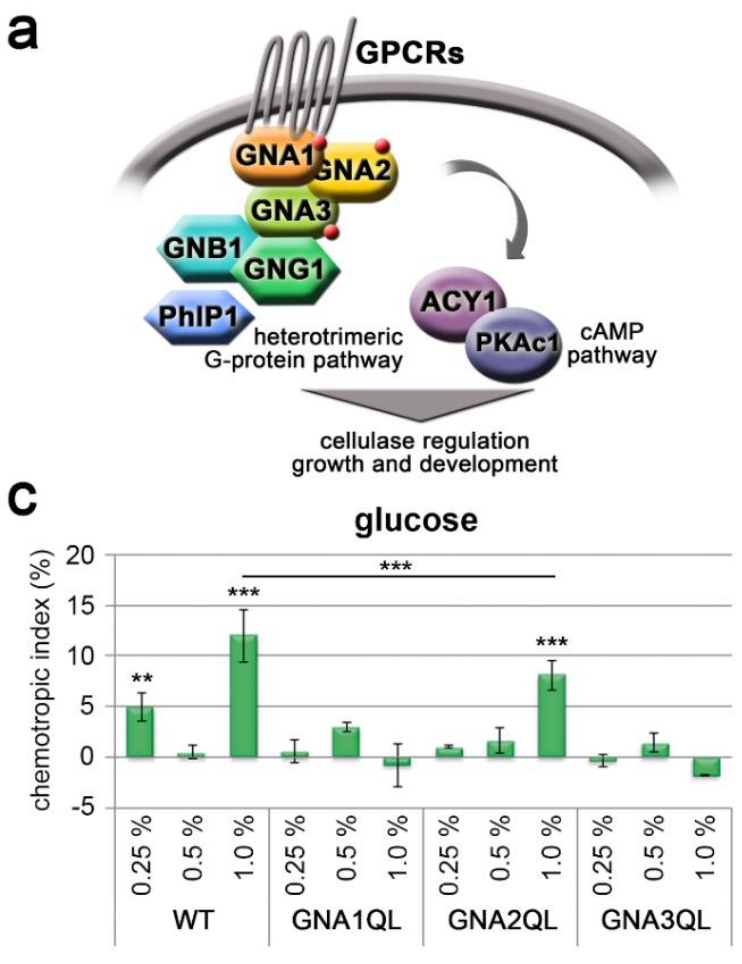

b

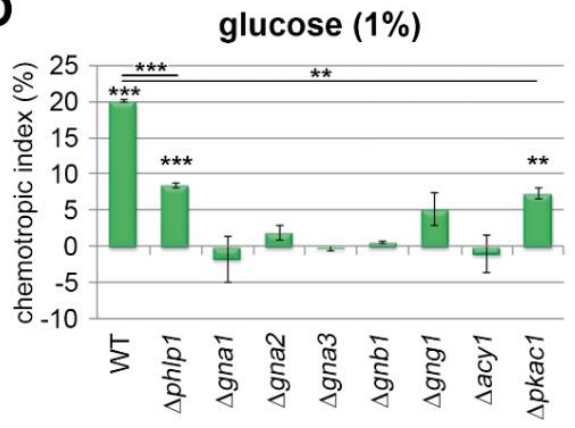

d

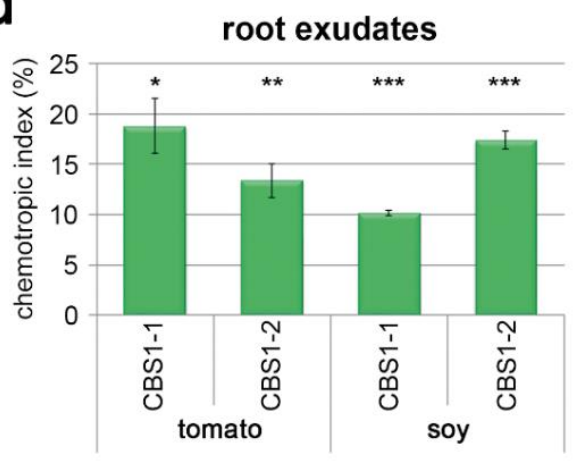

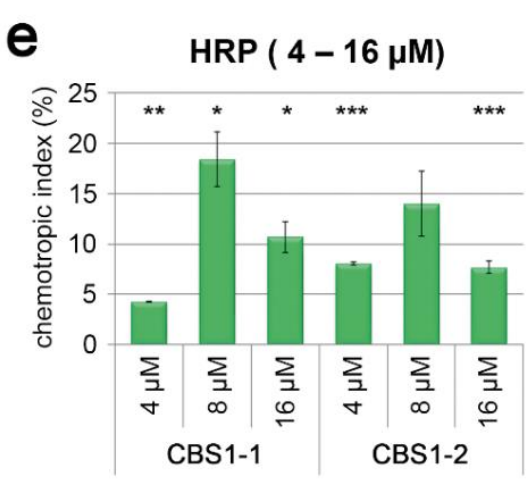

e

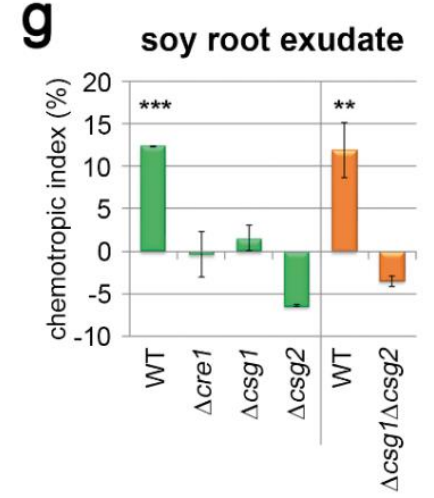

f

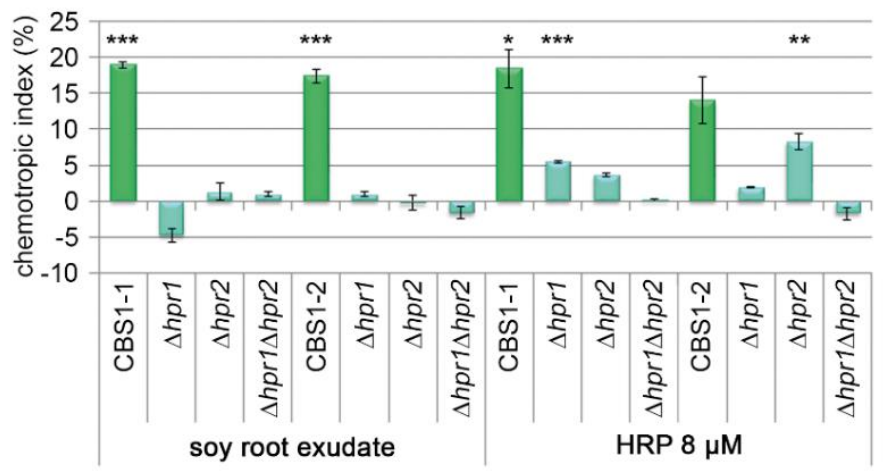

\section{h}

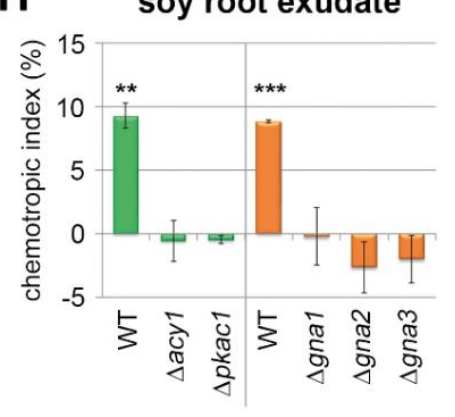

glucose

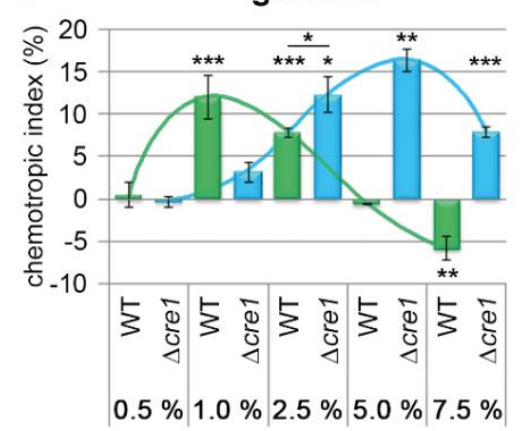

Figure 2. Glucose chemosensing requires the GPCRs CSG1/CSG2, heterotrimeric G proteins and the cAMP/PKA pathway. (a) Schematic representation of the heterotrimeric G protein-PKA/cAMP pathway in $T$. reesei. (b) Chemotropic response to $1 \%$ glucose of deletion mutants lacking the phosducin PHLP1, G alpha (GNA1/2/3), beta (GNB1) or gamma (GNG1) subunits, the adenylate cyclase ACY1 or the catalytic 
594 subunit 1 of protein kinase A (PKAc1). (c) Chemotropic response to different glucose concentrations of 595 strains carrying dominant activating alleles of the G protein alpha subunits GNA1, 2 or 3. (d) Chemotropic 596 response to root exudates of tomato or soybean plants, of the fully fertile strains CBS 999.97 MAT1-1 597 (CBS1-1) and CBS999.97 MAT1-2 (CBS1-2). (e) Chemotropic response to different concentrations of 598 horse radish peroxidase (HRP) of CBS1-1 and CBS1-2 . (f) Chemotropic response to soy root exudate or $5998 \mu \mathrm{M}$ HRP, of CBS1-1 or CBS1-2 or the single and double pheromone receptor deletion mutants derived 600 from these strains. (g) Chemotropic response to soy root exudate of deletion mutants lacking cre 1, csg 1 601 and/or csg2. (h) Chemotropic response to soy root exudate of deletion mutants lacking the indicated $\mathrm{G}$ alpha 602 subunits, adenylate cyclase (ACY1) or the catalytic subunit 1 of protein kinase A (PKAc1). (i) Chemotropic 603 response to different glucose concentrations of the wild type and the $\Delta$ cre 1 mutant. Error bars show standard 604 deviations from at least two biological replicates. Asterisks mark statistical significance of chemotropism 605 in comparison to the control (absence of a chemoattractant). Statistical significance between measurements 606 is indicated by asterisks over black bars. * p-value $<0.1$, ** p-value $<0.05$ and $* * *$ p-value $<0.01$. 

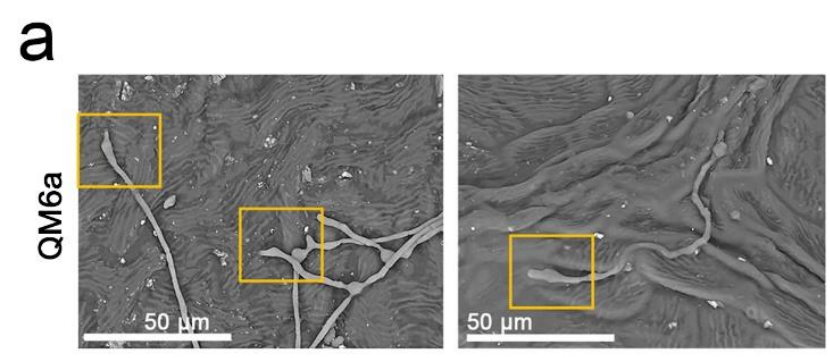

b
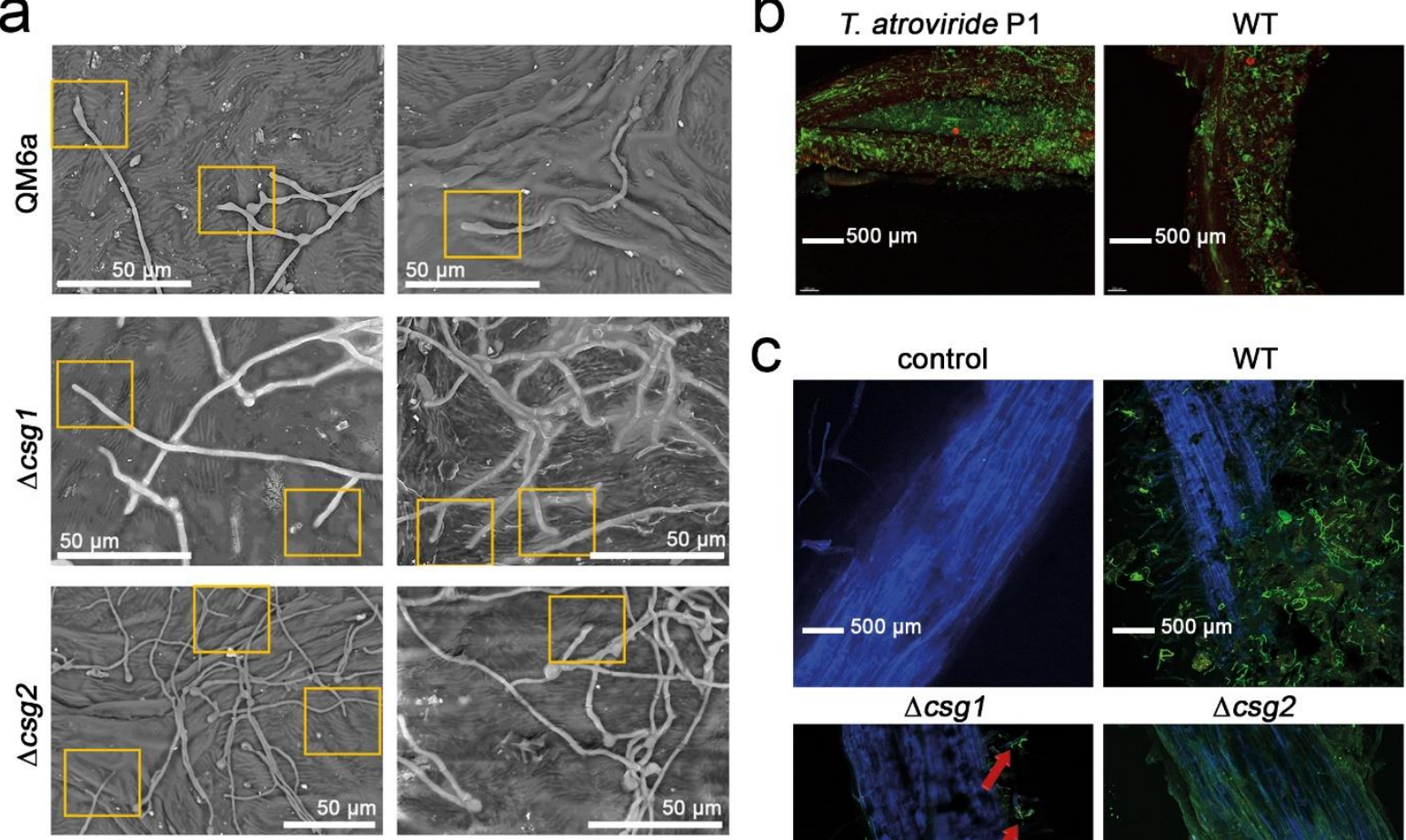

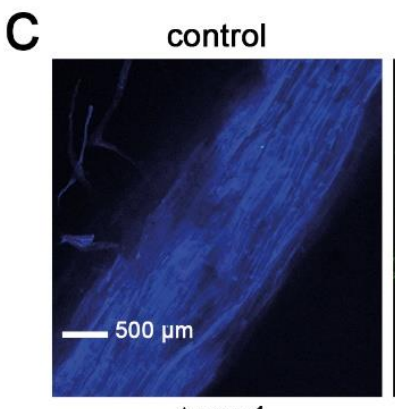

$\Delta \operatorname{csg} 1$

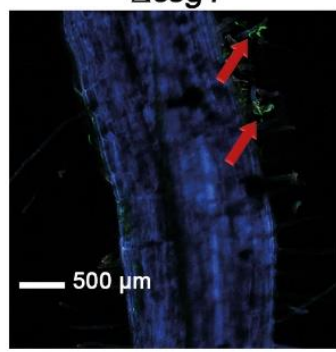

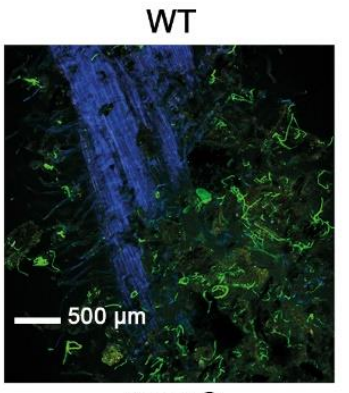

$\Delta \operatorname{csg} 2$

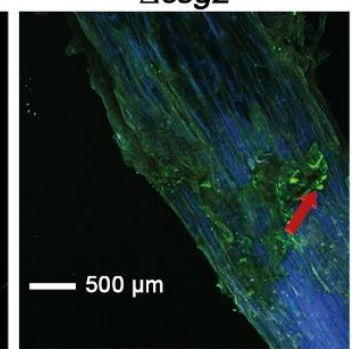

Figure 3. CSG1/CSG2 are required for developmental responses to plant surfaces. (a) Scanning electron microscopy analysis of interactions of QM6a and the receptor mutants $\Delta \operatorname{csg} 1$ and $\Delta \operatorname{csg} 2$ to a surface sterilized maple leaf. Yellow boxes indicate morphological alterations of hyphal tips in the wild type that are not observed in the deletion strains. (b) Confocal microscopy analysis of colonization of soybean roots by $T$. atroviride or T. reesei QM6a (WT). (c) Colonization of soybean roots by QM6a, $\Delta \operatorname{csg} 1$ or $\Delta c s g 2$. An uninoculated root is shown as control. 

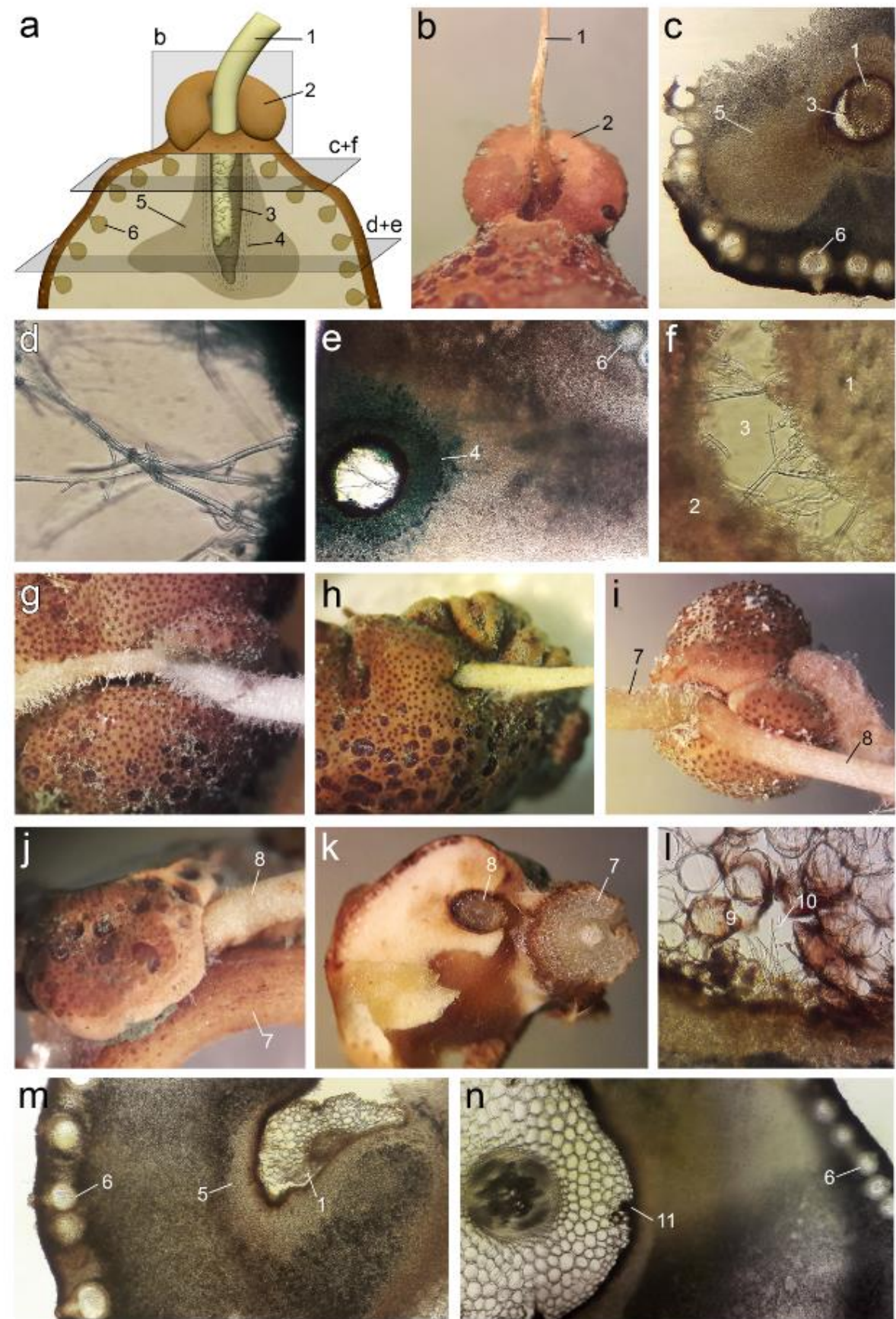

Figure 4. Fruiting bodies physically interact with soybean roots. (a) Schematic representation of the cross-sections through a fruiting body (FB) enclosing a soybean root [1]. Highlighted in grey are the macroscopic view of the entering zone (b), and two cross-sections (c) and (e) (200x) with the corresponding magnifications (d) and (f) (400x); Perithecia [6], zone in the FB showing a response to the soy root [5], methylene blue-stained pattern [4] and the cavity remaining after root tissue degradation [3]. (b) Example of a FB forming additional tissue [2] as reaction to the presence of the plant root [1]. (g-i) Macroscopic interaction between FB and roots; primary [7] and secondary roots [8].(j and $\mathbf{k})$ FB grown on the branching area of primary [7] and secondary root [8]. (l) Hyphae entering the root tissue [10] with root cells reacting to the fungus [9] are shown. ( $\mathbf{m}$ and $\mathbf{n}$ ) Cross-sections through FBs growing around roots with an entering point of mycelium [11] into the root tissue. Photos (b, $g-j, m$ and $n$ ) are composed of several merged pictures (focus stacking) for better depth of focus and in case of (m) and (n) for a broader overview of the whole cross-section. 


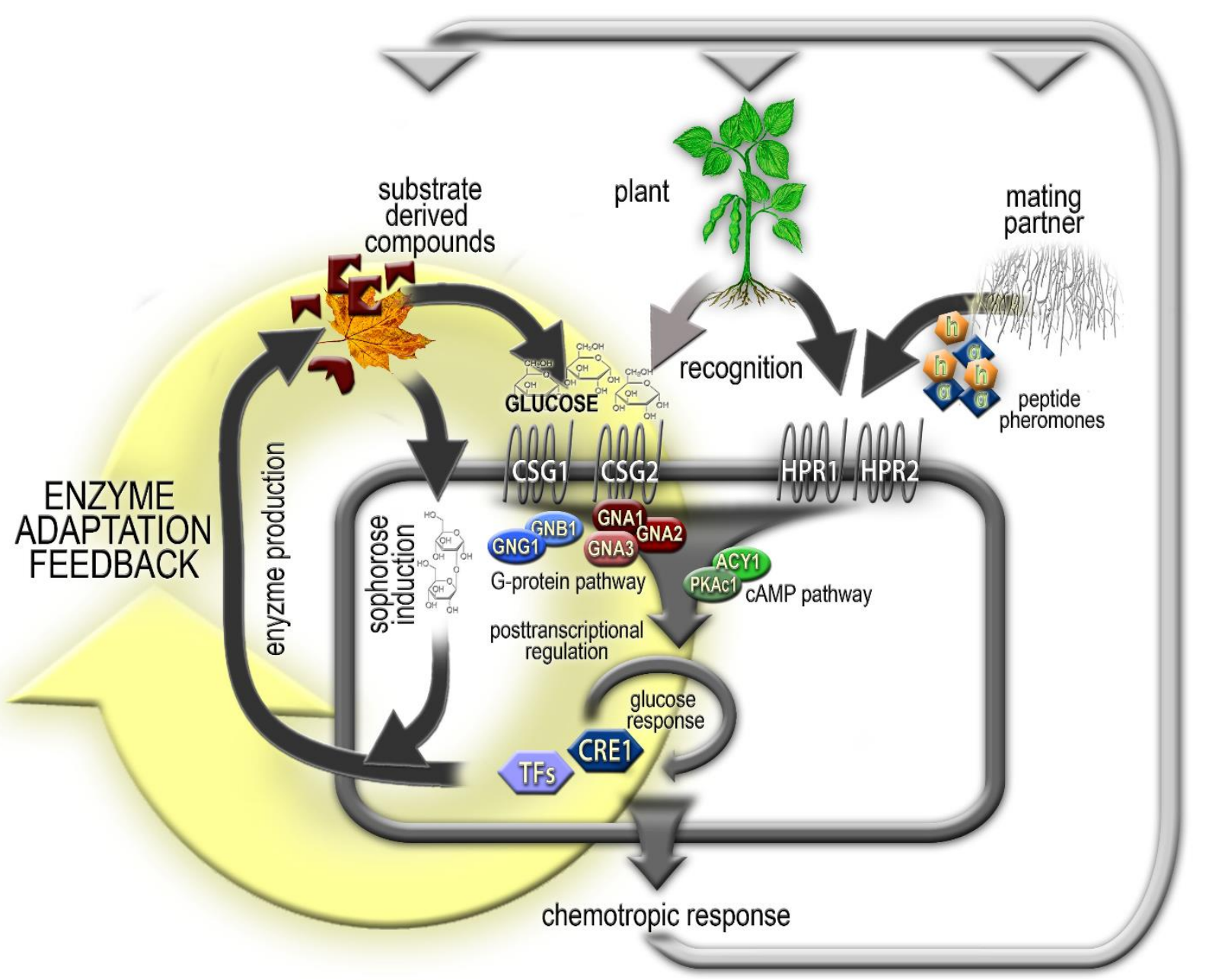

Figure 5. Integrative model of nutrient, plant and mating partner chemosensing in T. reesei. Transcription of genes encoding plant cell wall degrading enzymes (CWDEs) is triggered by the natural inducer sophorose. Moreover, CWDE biosynthesis beyond basal levels is initiated in response to defined levels of glucose liberated by enzymatic cleavage from plant biomass, requires the glucose sensor GPCRs CSG1/CSG2 and the heterotrimeric G protein-cAMP/PKA pathway and is negatively regulated by the carbon catabolite repressor CRE1 in response to high glucose levels. Proper nutrient sensing further results in morphogenetic responses and successful root colonization. Together these regulated pathways (enzyme secretion, glucose sensing, carbon catabolite repression and plant sensing) constitute an adaptation feedback loop which is used to distinguish between dead plant biomass (enzymatic degradation and glucose levels balanced) and living plants (enzymatic degradation not required for glucose liberation, no balance). The heterotrimeric $\mathrm{G}$ protein pathway integrates nutrient, plant and mating signals. Accordingly, transmission of nutrient signals by CSG1, CSG2 and CRE1 is required for proper plant recognition and sexual development is accelerated upon communication with the plant. 